\title{
Design Issues for a Robocup Goalkeeper
}

\author{
Riccardo Cassinis and Alessandro Rizzi \\ University of Brescia \\ Dept. of Electronics for Automation \\ Via Branze38, I-25123 Brescia - Italy \\ Phone +39-030-3715.469 - Fax+39-030-380014 \\ \{riccardo.cassinis, alessandro.rizzi\}@unibs.it
}

\begin{abstract}
This paper presents Saracinescu, the goalkeeper robot of the Italian team that was used at the Robocup '98 Paris championship. The machine features an original omni-directional vision system whose performance, enhanced by a simple but effective movement strategy, proved to be very smart and led to good results during the tournament. The paper describes the vision algorithms in detail, and discusses some issues that are still being developed and/or refined. An overview of the other components of the machine (mechanical structure and ball-kicking mechanism, computing architecture, auxiliary software routines for initial positioning, etc.) is also included.
\end{abstract}

\section{Introduction}

The Robocup championship offers a simple and well-structured environment, suitable for testing some innovative robot features like the visual guidance system presented in this paper. One of the simplifications that this environment introduces with respect to the real world is the small number of colors used in the playground and the rigid coding of their meaning. However, even if each game component has a unique color, problems for color matching still remain, due to illumination changes, shadows, etc.

The robot we present is the goalkeeper of the Italian team (ART), which exhibited a very good performance during the Robocup '98 Paris championship. Its main characteristics are an omni-directional vision system and a simple but effective reactive strategy.

The visual guidance system is based on color information grabbed with an omni-directional, quasi-spherical device. The intrinsic geometric complexity of the image is simplified by the use of color. Neither shape nor other geometric features are taken into account. Only the colors and the relative position of the objects surrounding the robot influence its movement. Even if the idea of using omni-directional visual devices had already been used in previous Robocup events, the presented one allows measuring not only the direction, but also the distance of relevant objects. The underlying idea has since then been adopted by several other researchers involved in the Robocup competition.

In Par. 2 it is presented the overall structure of the robot, the details of the vision subsystem are described in Par. 3, and in Par. 4 the strategy is introduced. 


\section{An overall robot design description}

The robot is based on a modified version of the widely used, commercial RWI Pioneer 1 platform. An on-board PC, with the appropriate power supply, was added to provide the necessary computing power. The PC (an Intel Pentium II) runs LINUX operating system, and is equipped with all the necessary peripherals, that include an Intel Video Recorder frame grabber used to acquire camera images, and a Wavelan wireless networking interface.

Given the task the goalie has to accomplish, the only possibility offered by the mechanical structure was to use the robot sideways, in order to make it able to quickly reach any point of the goal area. The original castor wheel that supports the weight of the robot was replaced with a spherical device in order to eliminate lateral skids when the robot reverses its movement.

A mechanical ball-kicking device has been mounted on the left side of the robot, that always faces the playground (Fig. 1). When the ball touches the lower edge of the kicker, it activates a mechanical switch that in turn triggers a "kick and reload" mechanism. Kicking power is provided by a steel spring, while reloading is accomplished by an electric motor. After each kick, it takes about two seconds to reload the mechanism. The kicking reflex takes place locally, i.e. with no computer intervention; the main computer can, however, disable and re-enable the device.

Another significant change was the addition of a large plexiglas pipe (Fig. 1), fixed on the upper part of the robot, that supports the mirror of the vision system, as it will be described in the following paragraph.
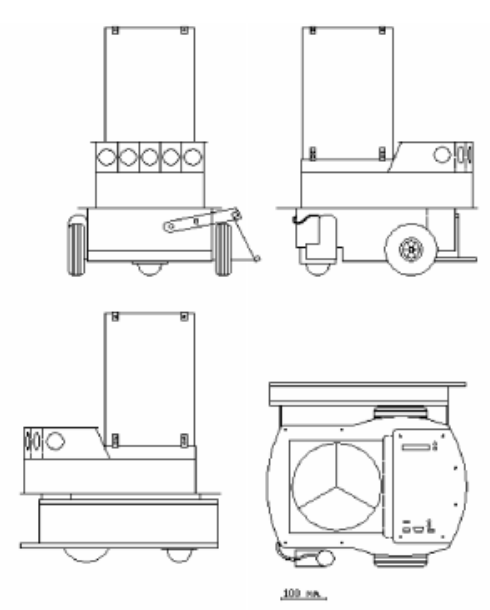

Fig. 1. Saracinescu structure design 


\section{The omni-directional visual device}

Several kinds of omni-directional visual sensors, with different geometrical characteristics $[2,3,4]$ and different optical pre-processing capabilities [1] have been investigated so far. They have been used for various robot navigation and selflocalization tasks.

The omni-directional device developed for Saracinescu uses a mirror with a spherical sector shape and an optical grade reflecting surface, that allows a clear vision of what is happening around the robot. The spherical shape of the mirror allows the perception of a larger amount of details in the area surrounding the robot and only rough visual information of the area away from the robot. The mirror axis is vertical, and the device (actually, a $20 \mathrm{~cm}$ diameter stainless steel pan lid was used) is supported by a clear Plexiglas pipe, that also houses the camera. The idea is to mimic the behavior of a real goalkeeper that does not care much about what is going on in the opposite half of the field, but pays great attention when the ball comes close him.

Fig. 2, besides giving an idea of the structure of the device, shows its most interesting feature: using a quasi-spherical mirror allows measuring not only the bearing of the bjects with respect to the robot axis, but also their horizontal elevation. Since all objects in Robocup lay on the ground and have known dimensions, the system can compute objects distance as well.

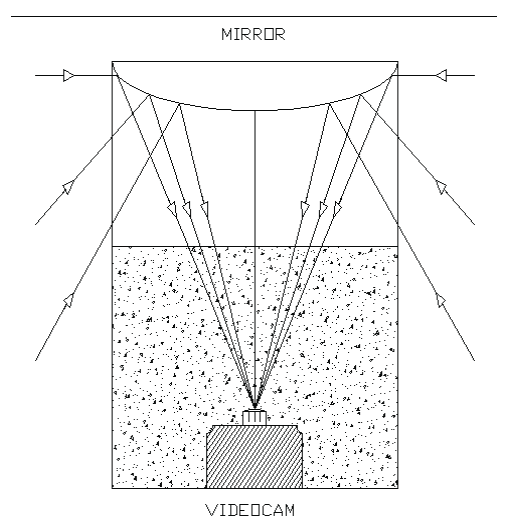

Fig. 2. The omni-directional vision system

An upward pointing CCD color camera grabs images reflected by the mirror. Its signals are processed in order to extract information about the goal posts and the ball. This information is used to keep the robot between the ball and the goal.

Fig. 3 shows an actual image grabbed from the camera. Grey scale rendering makes it hard to distinguish details, but it is quite easy to recognize the ball 
(red), the field walls and lines (white) and the goal (black, in the lower part of the image). The figure shows the results of processing superimposed to the original image: the recognized portion of the ball is drawn in blue, while the goal (originally yellow) is drawn in black. Careful analysis of the picture shows that all the field is shown, including the opposite goal. Far objects are poorly detailed but, as it was said, this is not important for the goalie.

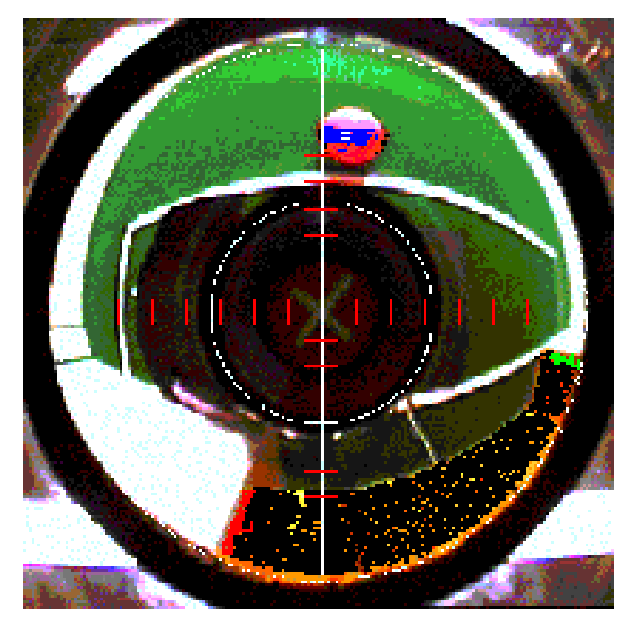

Fig. 3. An example of segmented omni-directional image

Measuring the Euclidean distance of objects from the center of the image allows estimating their distance from the robot. Since the exact shape of the mirror is not known, it would have been hard to determine the equation that gives actual distance as a function of the distance measured on the image. Instead, a look-up table was experimentally built, that lists correspondences between 50 and $230 \mathrm{~cm}$ (the estimated useful range), with $10 \mathrm{~cm}$ increments. The system uses linear interpolation for estimating intermediate values.

\subsection{Color segmentation and object recognition}

As it was said in the introduction, Robocup rules assign unique colors to all the objects in the playground. For this reason, no shape information is used for object recognition.

Two constraints influence the color segmentation process: the high computing speed required to effectively track the ball and the heavy varying illumination conditions. In fact, preliminary tests of the robot have been done on various training playgrounds, and have shown that apparent colors of the objects vary from field to field, due to the different light sources, materials used for building the field, etc. Additionally, our system has to deal with its very low-cost 
surveillance camera, whose color rendering and constancy are light-years far from perfection. Shadows produced by objects make the task even more tough.

To solve the problem of widely varying light conditions, a calibration phase has been introduced. During this calibration phase, usually performed once before each match, a supervisor has to select in the image a ball portion. The mean chromatic value among all the selected pixels is computed. This triplet is the center of the area in the RGB space that is clustered with a boundary threshold value for each chromatic channel. These boundary values are initially set equal to the relative chromatic variance of the selected pixels. This leads to a quite robust automatic clustering, but some problems still occur in presence of shadows and reflections.

Thus, a reinforcement of the color calibration phase has been introduced. In this second phase the supervisor selects a small area into which the ball is completely contained and the three RGB thresholds are incremented, thus enlarging the color clustering subspace, until a pixel that surely does not belong to the ball is selected.

After the calibration on the ball color, the same procedure is used to determine the color of the goal that can be alternatively yellow or blue. The whole procedure could be automated, but due to the strict deadline of the tournament, this has been deferred.

\subsection{Ball and goal-posts recognition}

During normal operation, the goal of the vision system is to recognize the ball and the goal posts, and to measure their position with respect to the robot. The speed requirements call for processing at least 10 frames per second, and some optimization has to be done to reach this goal.

The central part of the image does not contain any useful information, and is discarded. Only the external circular part of the image is considered for searching the ball and the goal-posts. With the mounted low-cost commercial camera, 5 bits per pixel for each chromatic channel have been used.

The color segmentation is performed using a region-growing algorithm which easily detects the ball. The goal posts are then detected selecting the boundary pixels between the goal color and the white field walls. Due to the geometry of the image, no confusion between the left and the right goal post can arise.

The ball is localized using the apparent center of gravity of the cluster of red pixels. To avoid localizing errors, a threshold on the minimum number of clustered pixels is used. The posts can be localized using the middle or the lower point of the connected boundary line. In the first case perspective errors due to the changing distance of the robot and the goal gate are introduced. In the second case the shadow of the robot can cover the lower post pixels introducing errors as well. The tests and the played matches proved that both methods work correctly in most practical cases. Regardless of the chosen method, what is really important for the robot movement, is not the goal-posts distance estimate, but their relative angles. 
The computing speed requirements, and the restrictions of the Pioneer software system, suggested that the ball and the goal recognition should be treated as two separate activities. Moreover, since the ball moves much faster than the robot, two ball searches are performed per each goal recognition.

\section{Strategy and robot movement}

All the strategies described in the following paragraphs have been implemented in $\mathrm{C}$ using the activities functionality of the Saphira programming environment which contains all the primitives for the robot movement. It was decided to avoid using Saphira behaviors because, even if they can help solving some of the problems, they add and unacceptable computing burden, making the robot too slow for any practical use.

Using Saphira activities and micro-tasks concept, different processes can be executed sequentially and cyclically within predefined time slices. In this way, various programs can run together with a simulated parallelism.

The basic strategy obviously is to keep the robot between the ball and the goal gate at all times. In order to do that, Saracinescu lays its right side toward the goal gate and moves back and forth like in Fig. 4. In this position the kicking mechanism faces the playground.

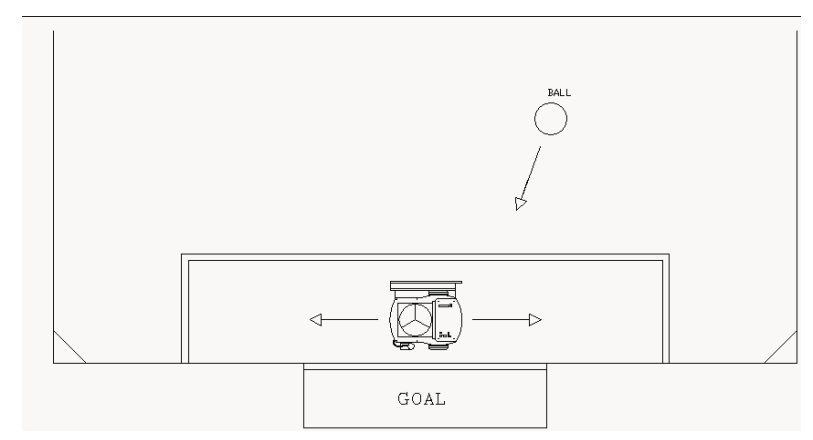

Fig. 4. Saracinescu basic strategy

Intercepting the ball only requires straight movements parallel to the goal: however, wheel skidding, encoder tolerance and crashes with other robots can result in involuntary robot rotations. To correct this problem, at regular time intervals, a process to control and correct the robot horizontal parallelism is executed.

Furthermore, since a constant angle between two reference points results in an arc, measuring the bearing of the goal posts does not allow determining the actual distance of the robot from the goal. Therefore, another task is started at 
regular time intervals. It controls the estimated distance of the goal posts and consequently moves the robot towards to or away from the goal.

Summarizing, there are four main logical activities running concurrently during normal robot operation:

1. grab the omni-directional image and extract distance and angle of the ball and the goal-posts,

2. keep the robot between the ball and the goal,

3. keep the robot parallel to the goal gate,

4. keep the distance between the robot and the goal constant.

All the geometrically derivable data are directly extracted from the image whenever is possible, introducing data redundancy. If, due to visual noise (occlusion, shadows, etc.) some data are missing, estimates from the data series support the robot for a limited time interval. After this period, if data are still missing, the robot enters a stall state until a sufficient amount of data is available again.

In order to obtain a not too "nervous" robot behavior and thus not to overstress the motors, a low-pass filter that averages data in time has been applied. It can be seen as an inertia increment that allows Saracinescu to keep a steady position without high frequency oscillation.

\subsection{The goalkeeping strategy}

The goalkeeping strategy is to maintain the line from the robot to the ball parallel to the bisecting line of the angle formed by the goal-posts and the robot (Fig. 5). In order to accomplish this strategy, this activity moves the robot only in the two directions parallel to the goal (Fig. 4).

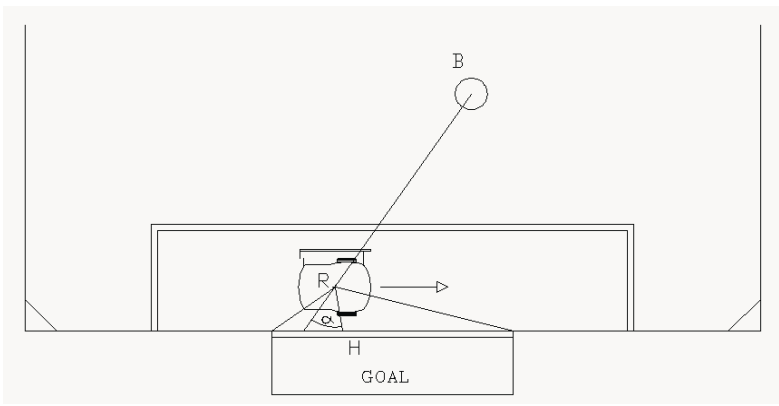

Fig. 5. The goalkeeping strategy

The bisecting line has been chosen instead of the median because it better protects the goal gate. The median would put the robot too close to the goal cen- 
ter. On the contrary, the bisecting line better protects the goal-post surroundings (Fig. 5), stopping also most of the bouncing kicks.

Moreover, this activity keeps the robot inside the small goal area monitoring the goal posts distance. Whenever the robot gets near a goal post, the main positioning algorithm is excluded and the robot starts to decrease velocity, so to stop at the goal-post point. Overtaking this point, in fact, is useless.

The parallelism keeping activity controls the angle between the robot heading and the goal line. The smallest angle the Pioneer can rotate is 5 degrees, thus angles below this value are not considered. Moreover, the Pioneer firmware has its own accidental rotation automatic corrector, so it is important to insert a delay before triggering this activity.

The goal distance control activity controls the robot distance from the goal. If the distance exceeds a threshold value, all the activities are stopped and a repositioning algorithm is executed. This algorithm drives the robot towards its default starting position like shown in Fig 6.

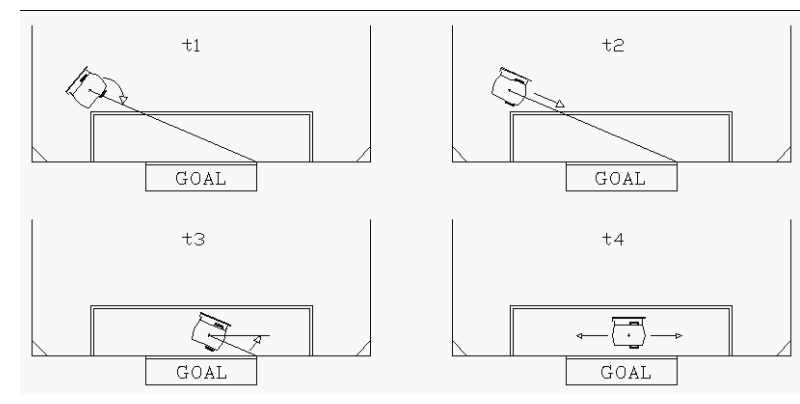

Fig. 6. The repositioning algorithm

\section{Conclusions and perspectives}

The performance of Saracinescu, that has been the official goalkeeper of the Italian Azzurra Robot Team at the Robocup 1998 championship in Paris, has gone beyond the most optimistic expectances. Its reactive behavior lacks any reasoning and forecast capability, but the machine was perfectly apt for the simple task it had to accomplish. The robustness of the vision system was however a determining factor for its success. It localized the ball during all the matches with good precision and regardless of the illumination, of the shadows and of reflections in the playground. This suggests that all the robots in a team, and not only the goalkeeper, should use a similar system for visual data acquisition.

It can be expected, however, that the performance of other robots will increase in future championships, and that several improvements should be made 
to the structure of our goalie. Besides the mechanical requirements, that suggest completely rebuilding the machine in order to have a much lighter and faster robot, it would be desirable to fully automate the color calibration procedure, using an automatic ball and goal-post detection. Object recognition should be performed using predictive algorithms, in order to speed up the process. Recognition of robots should be introduced, in order to allow reasoning about where the ball will be kicked by an opponent robot, and how it should be usefully passed to a fellow.

\section{References}

1. R. Cassinis, D. Grana, A. Rizzi, Using Colour Information in an Omnidirectional Perception System for Autonomous Robot Localization. In Proceedings of EUROBOT96 First Euromicro Workshop on Advanced Mobile Robots '96, Kaiserslautern (Germany), 1996

2. Y. Yagi, H. Okumura, M. Yashida. Multiple Visual Sensing System for Mobile Robot. In Proceedings of the IEEE Int. Conference on Robotics and Automation, 1994, Vol 2, pp. 1679-1684

3. Y. Yagi, Y. Nishizawa, M. Yashida. Map based Navigation for a Mobile Robot with Omnidirectional Image Sensor COPIS. In IEEE Trans. Robotics and Automation, Vol.11, No 5, 1995

4. K. Yamazawa, Y. Yagi, M. Yachida. Omnidirectional Imaging with Hyperboloidal Projection. In Proceedings of the IEEE/RSJ IROS'93, Vol. 2, pp. 1029-1034, 1993 